\title{
Entre science et politique, la criminologie, une science sociale en balbutiements...
}

Martine Kaluszynski*

https://orcid.org/0000-0002-8951-1389

Sciences de l'homme, sciences du social, sciences du politique

Les sciences de l'homme sont requises, particulièrement depuis le XIX ${ }^{\text {ème }}$ siècle, pour donner à voir "scientifiquement" le social ${ }^{1}$. Mais la mobilisation des savoirs se fait de telle façon, la relation est souvent si symbiotique entre la production des connaissances et ses contextes sociaux, économiques, politiques, culturels, que ce que ces savoirs donnent finalement à voir ce sont d'abord les préoccupations plus ou moins conjoncturelles qui les suscitent. Sciences sur le social, elles deviennent sciences dans le social, manifestation privilégiée du social dans lequel elles sont finalement immergées. La criminologie au XIX ${ }^{\text {ème }}$ siècle est de ce point de vue absolument exemplaire.

Sciences sur le social, elles deviennent sciences dans le social, manifestation privilégiée du social dans lequel elles sont finalement immergées. La criminologie au XIX ${ }^{\text {ème }}$ siècle est de ce point de vue absolument exemplaire.

Sous le nom de crimes et délits, on juge toujours bien des objets juridiques définis par le code, mais en juge en même temps des passions, des instincts, des anomalies, des infirmités, des inadaptations, des effets de milieu ou d'hérédité (Foucault, 1975, p. 22).

* Institut d'Études Politiques, Grenoble, França.

1. Je voudrais ici remercier vivement Marcos Cesar Alvares pour sa confiance et Mariana Chies Santiago Santos pour sa relecture attentive et ses commentaires pertinents. 
Economiquement, socialement, culturellement, la fin du XIX ${ }^{\text {éme }}$ siècle est une période riche et dense. La croissance industrielle et l'urbanisation ont bouleversé les modes d'existence, déstabilisant une frange importante de la population. La crise économique des années 1880 a aggravé cette réalité sociale déjà précaire.

Au pouvoir, en France, la jeune Troisième République prône des valeurs d'ordre, de stabilité, de travail, et a la volonté de tout mettre en œuvre afin de les faire respecter. A la révolution technologique et industrielle s'ajoute une rare floraison de disciplines due tout autant à la qualité de la production scientifique, littéraire ou artistique, qu’à sa variété, ses contrastes et ses contradictions...

Siècle de la misère ouvrière et des grands tumultes, de la passion et du foisonnement des idées, la violence est perçue comme négative: le crime, la criminalité sont les terrains privilégiés pour refléter les inquiétudes, les peurs d'une société en mouvement. Angoisses qui dépassent le stade même du crime, le sentiment "d'insécurité" économique et social ne faisant que se déplacer vers le pôle visible qu'est le monde du trouble et du désordre. L'homme moderne veut tout maîtriser, rationaliser, même si dans ce domaine l'irrationnel côtoie souvent le rationnel, voire tend à l'emporter.

C'est dans ce contexte qu'apparaît l'anthropologie criminelle ou la criminologie, et on ne peut nier la valeur du lien entre cette période riche en "recherche", et ce savoir "scientifique" traitant du désordre, dont Michel Foucault parlait ainsi dans les premières pages de Surveiller et punir:

L'expertise psychiatrique, mais d'une façon plus générale l'anthropologie criminelle et le ressassant discours de la criminologie trouvent là une de leurs fonctions précises: en inscrivant solennellement les infractions dans le champ des objets susceptibles d'une connaissance scientifique, donner aux mécanismes de la punition légale une prise justifiable non plus seulement sur les infractions, mais sur les individus; non plus sur ce qu'ils ont fait, mais sur ce qu'ils sont, seront, peuvent être. Le supplément d'âme que la justice s'est assuré est en apparence explicatif et limitatif, il est en fait annexionniste [...].

Depuis 150 ou 200 ans que l'Europe a mis en place ses nouveaux systèmes de pénalité, les juges, peu à peu, mais par un processus qui remonte fort loin, se sont donc mis à juger autre chose que les crimes: "l'âme" des criminels. [...].

[...] Une autre vérité a pénétré celle qui était requise par la mécanique judiciaire une vérité qui, enchevêtrée à la première, fait de l'affirmation de culpabilité un étrange complexe scientifico-juridique (Foucault, 1975, p. 24).

La criminologie sera un merveilleux instrument de connaissance de cette fin du $\mathrm{XIX}^{\text {ème }}$ siècle, dont il cristallise les enjeux, les peurs, les valeurs. Une période dont on voit bien que la menace ou le risque d'êtres dangereux ou monstrueux va fonder des 
actions de préservation, de prévention sociale, de rejet ou de relégation, réagissant en médecin dans ses analyses sur la société (parlant de pathologie, de sélection, de corps, de prophylaxie) et agissant en juriste dans ses actions. La criminologie construit, reflète et participe à cette société qui l'a vu naître. A travers ces écrits on retrouve des menaces d'ordre multiple: la peur du vol, de l'enfant criminel; une peur propriétaire, une peur adulte, avant tout la peur du désordre, de ce qui est différent ou contre nature.

L'histoire de la criminologie au XIX ${ }^{\text {èe }}$ siècle est à la fois une sociologie du statut social des sciences humaines et une histoire politique et constitue bien ainsi une contribution, non pas seulement à une histoire des sciences de l' homme comme une histoire des connaissances d'un domaine particulier, mais à un travail de dévoilement des conditions socio-politiques et culturelles de reproduction des connaissances, ici celui de la société républicaine de la fin du XIX ${ }^{\text {ème }}$ siècle.

Un savoir, des techniques, des discours scientifiques se forment, s'enrichissent, se nouent... en adéquation avec une société pour qui la politique sera fondée sur la connaissance. Les enjeux, dès lors sont ceux d'une refonte de la légitimité politique à partir de la compétence (Dammame, 1995) et ce savoir scientifique va épauler la décision politique dans la mesure où il donne à l'Etat le moyen, (l'illusion) de comprendre et de pouvoir agir au mieux. Il existe une infinité d'approches et de compréhensions en ce qui concerne la criminologie, qui en fait un objet délicat à cerner, et dont il faut dresser avec ampleur la représentation. Est "criminologue", "criminologiste" ou "criminaliste", praticien de la "criminologie"3 celui qui parle, écrit, de manière argumentée, logique, scientifique du crime et du criminel. Cette diversité est représentée à travers une abondante littérature sur ce thème, tout au long du XIX ${ }^{\text {ème }}$ siècle. De multiples définitions recouvrent ce terme de criminologie. On peut retenir: l'étude scientifique des causes et conséquences du comportement antisocial, étude qui tente de mettre en place solutions et remèdes. On peut citer celle très générale qu'en fit $\mathrm{E}$. Durkheim:

Nous constatons l'existence d'un certain nombre d'actes qui présentent tous ce caractère extérieur, que, une fois accomplis, ils déterminent de la part de la société cette réaction particulière qu'on nomme la peine. [...]

Nous en faisons un groupe sui generis, auquel nous imposons une rubrique commune: nous appelons crime tout acte puni et nous faisons de crime ainsi défini l'objet d'une science spéciale: la criminologie (Durkheim, 1912).

2. Selon les termes de l'époque, sans aucune réelle distinction entre tous ces mots.

3. Bien que le terme n'existe pas de suite en tant que tel, et que l'idée s'est perpétuée sous le nom d'anthropologie criminelle. 
Cette diversité de compréhension et d'approche fait de la criminologie un objet délicat à saisir, porteur d'une trop large représentation de sens ${ }^{4}$.

Nous voudrions ici précisément montrer la dimension sociologique de ces écrits portés plutôt par des médecins, quelques juristes intéressés par le social et par l'impact de leurs travaux sur les possibles transformations du monde. Une dimension silencieuse, discrète car non institutionnalisée mais qui porte en elle tous les aspects d'une réflexion montrant à quel point la criminologie est une science de l' homme et une science du politique donc une science sociale en construction, une science sociale qui devient un savoir qui fonde le politique "requalifiant le champ des compétences de l'Etat" (Procacci, 1995, p. 413). Nous allons revenir aux fondements de ce que fut la sociologie du crime et donc la sociologie (du droit) pénal(e) en s'attachant aux débuts de la criminologie en France et tester ses rapports à la "sociologie", rapports de grande proximité et d'inspiration concrètes.

Nous reviendrons sur les fondements de l'anthropologie criminelle, en faisait un détour par l'aspect sociologique de l'école Italienne à travers Lombroso mais surtout Enrico Ferri et l'école française autour de Lacassagne, et sa grande figure juriste et sociologue que fut Gabriel Tarde, traquant dans la forme, les traces, l'esprit, l'empreinte sociologique d'un savoir qui ne doit pas seulement à son caractère anthropologique, malgré son nom, et qui permet de montrer la marque sociologique naissante et la part de naturalité dont la sociologie a été marquée dès ses origines. Analyseur du politique et du social, ce discours sur l'homme criminel est avant tout un discours sur l'homme, dans un de ses états exacerbés qu'est le crime, mais un discours sur l'homme et la société.

Genèse d'un savoir expert: les débuts de l'anthropologie criminelle

C'est au XIX ${ }^{\text {ème }}$ siècle, dans les années 1880, qu'apparaît l'anthropologie criminelle ou la criminologie. Le crime est au XIX ${ }^{\text {ème }}$ siècle la "figure offensive de la monstruosité" par excellence (Tort, 1985). Le crime, la criminalité sont des terrains privilégiés pour refléter les angoisses et les peurs d'une société en construction. De Cesare Lombroso à Alexandre Lacassagne, on aura affaire à un mélange savant du biologique et du social et d'un discours qui se nourrit du positivisme de Comte, des théories pasteuriennes, de l'interpsychologie de Tarde, du contingentisme de Boutroux.

4. C'est également le cas pour le crime à propos duquel Manouvrier dans L'innéité criminelle, conférence posthume 1903, explique bien la profusion de sens que peut recouvrer ce terme, p. 14.

5. Pour ce texte, j'ai repris et me suis appuyée sur plusieurs de mes travaux déjà réalisés. L'idée étant de proposer et donner à voir la genèse d'un savoir très anthropologique mais proche de la sociologie en balbutiements. 
Aux origines d'une criminologie française, se retrouve la criminologie italienne personnifiée par C. Lombroso, E. Ferri, R. Garofalo, elle-même liée à une pensée criminologique antérieure, ou des hommes comme C. Beccaria, A. Lavater, F. Gall ont joué un rôle prépondérant. A une époque tournée vers la science et vers l'homme, des méthodes, des techniques nouvelles apparaissent quasi simultanément avec les idées en germe dans les esprits curieux ${ }^{6}$. C'est donc à une paternité éclatée que peut se référer la criminologie, dont on peut néanmoins privilégier deux éléments. Au niveau des instruments, l'apparition des premières statistiques judiciaires françaises est un fait important ${ }^{7}$. C'est à la Restauration où la grande poussée de délinquance des années 1815-1818 inquiète que s'établit au Ministère de la Justice une direction des statistiques qui publie dès 1825 Le Compte Général Administratif de la Justice Criminelle $(\mathrm{CGAJC})^{8}$ Annuel dès cette année, ininterrompu jusqu'à nos jours, le CGAJC sera au fondement de nombreuses études criminologiques. En ce qui concerne hommes et savoirs confondus, citons D'Angeville ou les travaux de science morale de Guerry mais aussi la phrénologie de Gall ou la physiognomonie de Lavater qui tente de "juger la personne intérieure par l'extérieur", et se propose de connaître ainsi les signes sensibles de nos forces et de nos dispositions naturelles; sans oublier Quetelet, qui avec sa Physique sociale ou essai du développement des facultés de l'homme veut découvrir les "Lois" d'après lesquelles l'homme se reproduit et croît. Les faits seraient soumis à certaines lois de périodicité comme les phénomènes les plus mécaniques de la nature. Quetelet arrive à la constatation qu'il existe une constance du fait criminel qui obéit à des lois et à l'existence d'un penchant au crime. Ce qui ressort fondamentalement de ses travaux, c'est l'idée que désormais l'homme est susceptible d'une approche scientifique, quantifiée.

\section{La dimension "sociologique" de Lombroso et de l'Ecole italienne}

Cesare Lombroso va formaliser le premier les orientations de cette nouvelle approche avec l'anthropologie criminelle, unissant la statistique et l'anthropologie. Il impose l'idée que la cause profonde de la délinquance se trouve dans les anomalies corporelles et mentales, que les criminels sont biologiquement arrêtés dans l'évolution. Il existe des criminels-nés, déterminés à le rester. Le déterminisme est la clé de voûte

6. Voir G. Leclerc, 1979.

7. Voir l'article fondateur de M. Perrot "Délinquance et système pénitentiaire en France au XIX ${ }^{\text {ème }}$ siècle", 1975; Perrot, "Premières mesures des faits sociaux, les débuts de la statistique criminelle en France, 1780-1830”, 1976.

8. Voir Perrot et Robert Compte Général de l'Administration de la Justice Criminelle en France pendant l'année 1880 et Rapport relatif aux années 1826 à 1880, 1989. 
de cette pensée dont un aspect révolutionnaire est de s'intéresser au criminel, à sa personnalité alors que jusque-là seul le crime était l'objet de préoccupations. L'alliance intellectuelle avec E. Ferri, R. Garofalo, nuancera et enrichira cette pensée à la fois luxuriante et rigide. Ces théories extrêmement séduisantes, puisqu'apportant une réponse scientifique et rationnelle à l'explication de l'acte criminel, entraîneront néanmoins après un temps d'enthousiasme, des critiques sur la méthode et les principes. Néanmoins, ces polémiques n'abattront pas le jeune savoir au stade de balbutiement, il créera au contraire un regain dans cette jeune discipline et si un des héritages les plus redoutables de Cesare Lombroso est d'aboutir à une pensée où les criminels constituent une véritable race à part, avec des stigmates de structure précis, biologiques ou psychologiques qui constitueraient une sorte de marque instinctive et indélébile. Un des aspects extrêmement positifs de ces théories est d'avoir imposé l'étude de l'homme délinquant, déplacé le regard du crime vers le criminel et en cela, bouleversé les conceptions du monde pénal, juridique et policier.

\section{Des méthodes "ethnologiques"chez Lombroso}

Lombroso et le type criminel

Les méthodes ou techniques sont celles en usage à cette époque et étroitement liées à la formation même de celui qui les préconise: un médecin (militaire) spécialisé en médecine clinique, anthropométrique (surtout sur les cadavres), à l'autopsie, à l'étude des crânes. Lombroso a également travaillé avec et sur les photographies. Il s'est appuyé pour cela sur les expériences faites suivant le système de Galton (1878, p. 33). Il se prévaut du résultat de certaines photos composites, et utilise l'étude de photographies des criminels pour contrôler et fixer la fréquence du type physionomie du criminel. Pour lui, la photographie confirme à quel point le type ethnique s'efface chez les criminels, tandis que beaucoup offrent entre eux une véritable ressemblance (Lombroso, 1887, pp. 231-234). La photographie révèle également pour Lombroso la fréquence de l'aspect féminin chez quelques voleurs et chez les pédérastes, et de la virilité chez beaucoup de femmes criminelles, surtout les meurtrières ${ }^{9}$.

9. G. Tarde attaquera sur le procédé utilisé. C. Lombroso (Tarde, 1972, pp. 220-221). “Fusionnez par le procédé de Galton 10 ou 12 photographies de Chinois, vous obtiendrez un portrait générique où, sous leurs différences effacées, leurs similitudes apparaîtront seules en un relief singulier, vivante abstraction, incarnation individuelle de la règle idéale dont les individus sont les déviations oscillatoires. Cette image-type a cela de particulier qu'elle embellit ce qu'elle combine et qu'elle explique ce qu'elle résume." 
C. Lombroso ajoute à ce "type criminel" une étude sociale. Il va rechercher dans la religion des criminels, leur intelligence et leur degré d'instruction, les caractères de l'argot, leur écriture, leur littérature, leur art et leur industrie, ces signes biologiques et psychiques destinés à compléter le type anatomique exposé précédemment.

Prenant en compte les aspects sociologiques du phénomène criminel, principalement sous l'influence d'E. Ferri ou R. Garofalo, Lombroso étaye une nouvelle conception pénale pour ces délinquants, désormais "connus", chiffrés, classifiés. Il a mis en avant que le crime est le fait d'un homme et n'existe pas en dehors de lui. Connaître le crime, c'est d'abord connaître le criminel, en cela Lombroso s'élève contre l'école pénale dominante qui pense que l'infraction est une entité juridique qui relève de la logique du droit et de la spéculation

Au lieu de prendre le délit comme une donnée dont part le criminaliste, Lombroso veut y aboutir après en avoir déterminé les causes, il entend le faire par l'observation, le message, l'expérimentation scientifique ${ }^{10}$. Ainsi, outre les explications biologiques et physiologiques sur le criminel, Lombroso a réfléchi sur les implications pénales résultant de ces conceptions. C'est une partie de ses travaux beaucoup plus enfouie et pourtant intéressante. De la peine capitale vu un procédé complémentaire de la sélection naturelle. La question de la peine capitale donna l'occasion à Lombroso et à ses disciplines de réaffirmer leur conviction de la nature innée de la transgression chez les criminels-nés. "L'atavisme nous fait comprendre l'inefficacité de la peine envers les criminels-nés et le fait de leur retour constant et périodique (au crime)" (Lombroso, 1899, p. 448).

Il existe, il est vrai, écrit Lombroso, un groupe de criminels, nés pour le mal, contre lesquels viendraient se briser comme contre un écueil toutes les cures sociales, ce qui nous contraint à leur élimination complète, même par la mort (Lombroso, 1899, p. 543).

A propos de la prison, Lombroso est assez catégorique. Il repousse le régime cellulaire. Il voudrait que les détenus rendus à la liberté soient actifs et repentis après

10. M. Ancel, Le centenaire de L'Uomo Delinquente, RSC, 1978, II, p. 288: "En réclamant et en pratiquant une étude scientifique positive de ce délinquant (et par là de tout le phénomène criminel), et en proclamant que cette étude relève désormais des sciences de l'homme, Lombroso récuse par là même le système classique fondé sur une métaphysique du droit, immuable dans le temps et dans l'espace ; et c'est ainsi qu'il se révolte contre la responsabilité fondée sur le libre arbitre, la peine proportionnée au délit et la sanction rétributive. Son ouvrage opère une remise en cause du système pénal traditionnel et de ses bases fondamentales". 
un traitement particulier et différent selon chaque catégorie ou classe de criminels. Si Lombroso est résolument adversaire de la prison, c'est qu'elle est pour lui un des grands facteurs de crime, une monstrueuse absurdité. "Nous croyons défendre et venger la société en emprisonnant les criminels et nous leur fournissons au contraire les moyens de s'associer et de s'instruire réciproquement dans le mal et même d'y trouver de vraies jouissances." (Lombroso, 1889, p. 265).

Lombroso dénonce donc les effets intimidants et l'action néfaste que peut avoir la prison. Il va se tourner vers les peines extra-pénitentiaires. Il faut éviter les peines de prison courtes et répétées et se tourner plutôt vers des peines légères qui frappent la vanité, comme certaines peines corporelles: Le fouet, pour les délits légers, mais également la mise aux arrêts chez soi, l'admonition judiciaire, l'amende, le travail forcé en liberté. Pour les aliénés criminels, il préconise la création d'asiles criminels dotés d'un personnel spécialement choisi et rémunéré en fonction de la mission. Enfin, il voudrait voir disparaître toute pénalité pour un certain nombre de délits comme l'avortement ou même l'infanticide. Il reste modéré pour les délits propres aux criminels d'occasion (vols d'aliment, diffamation, duel ou adultère).

\section{Les causes sociales du crime chez Lombroso}

Afin de parfaire un portrait et une étude déjà largement entamés, après les causes organiques et individuelles, Lombroso s'attache aux causes sociales de la criminalité. L'alcoolisme joue pour Lombroso un rôle prépondérant dans la genèse du crime. L'alliance constante de l'alcoolisme et du paupérisme est un argument précieux. Bien que Lombroso combatte cette vieille idée que la misère mène au crime, il concède bien volontiers que le manque de pain incite au vol, et que l'alcoolisme, l'anémie, la scrofule qui sont les inéluctables compléments de la disette, facilitent la dégénérescence et sont le fumier sur quoi fleurira le crime. Mais pour lui, la richesse lui apparaît comme un facteur presque aussi notable que la criminalité. C'est que, pense-t-il, la richesse est une source de dégénérescence pour d'autres causes, comme la syphilis et l'épuisement; c'est qu'elle pousse au crime par la vanité, pour surpasser les autres par le terrible besoin de paraître, de faire figure dans le monde. En outre, là où la richesse est grande, l'agglomération se produit, amenant ainsi un foyer favorable à l'éclosion de la délinquance. Le maître italien conclut que les milieux riches sont les plus dangereux, non seulement pour ce qu'il appelle les criminels-nés, mais même pour les criminels d'occasion.

A cet effet, autre paradoxe non moins fort mis en lumière par Lombroso, c'est que le progrès de l'instruction favorise le progrès de la criminalité. Les bandes internationales sont formées de gens dont l'instruction est extrêmement supérieure 
à celle de la moyenne des policiers. Ils possèdent souvent pour certains d'entre eux des notions fort claires de ce qu'est la police scientifique, qu'un Reiss ou un Hans Gross ou un Bertillon commencent à peine à répandre. Il est vrai que la connaissance la plus parfaite de la technologie et des progrès de la science n'accroît pas d'un seul coup la valeur morale de celui que les acquiert. Le développement intellectuel est indépendant du degré de clarté de la conscience. Ainsi, un criminel instruit n'en est que plus dangereux parce que savoir est une force, non une vertu.

On retrouve dans ces causes l'influence non douteuse del'hérédité, un des éléments le plus solidement établi dans la genèse du crime; également les causes géographiques, l'influence de mauvais gouvernements, l'effet de la prédominance d'une classe, le rôle des sectes, les crises économiques, voire le parlementarisme dont Lombroso fait un procès en règle et désigne comme facteur de crimes ${ }^{11}$. Malgré cette consciencieuse étude du facteur social, Lombroso n'a pas abandonné ses idées anciennes. Le facteur individuel reste pour lui l'élément primordial, l'essence de la criminalité.

Lombroso pense qu'il y a des conclusions et des solutions au point de vue de la prophylaxie et de la thérapeutique du crime. Il est par cela même partisan convaincu de ce qu'on appelle "les substitutifs pénaux", système qui en appelle à la création de conditions sociales et de bases législatives susceptibles de priver le crime de certains de ses éléments qui en favorisent le développement". Un système qui tente de supprimer le crime, en supprimant l'occasion. Quelques exemples concrets: la distribution de combustibles aux paysans supprime le vol de bois mort, la suppression des douanes fait disparaître les contrebandiers, les maisons de tolérance amènent la diminution de la séduction et des viols, le divorce tend à réduire le nombre d'adultères, etc.; sans compter les progrès de la police, moyen à la fois préventif et curatif d'agir sur le crime. On peut ajouter pêle-mêle à ces conceptions d'autres projets que Lombroso avait en tête: - l'introduction d'avocats spéciaux pour les personnes pauvres, - une procédure simplifiée en matière d'amende, - l'abolition du jury populaire, - l'attribution du Procureur public du droit de demander des dommages et intérêts en faveur de la victime.

11. "Le parlementarisme qui a été appelé avec raison la plus grande des superstitions modernes fait perdre de vue aux députés les hautes visées de l'État, en les poussant à couvrir de l'irresponsabilité, même devant le délit, quelques élus qui deviennent pour cela des criminels par occasion, s'ils ne le sont pas déjà par la naissance. De nos jours, le roi vient assurément en seconde ligne, mais les 700 pseudo-rois qui nous gouvernent sont d'autant plus violents et dangereux qu'ils sont cachés et font entrer l'injustice par tous les pores de la nation jusque dans les vallées les plus reculées qui ont le malheur de posséder un représentant. Nous avons lutté pendant des siècles pour abolir les privilèges des prêtres, des guerriers et des rois et maintenant nous maintiendrions sous l'étrange prétexte d'une prétendue liberté des privilèges extraordinaires, tel que celui de commettre des crimes de droit commun, à plus de 700 rois." (Lombroso, cité par E. Locard, revue critique, AAC, 1907, pp. 275-276). 
Ainsi Lombroso qui agit en naturaliste dans ce monde juridique évoque une nouvelle approche du droit pénal, non point la punition d'une faute morale mais la sanction, basée sur le droit que la société revendique pour se défendre; non point le libre arbitre, mais le danger potentiel que le criminel constitue. En fait, sont mis en relief des critères différents de détermination de la sanction pénale, basés sur la personnalité du criminel plutôt que sur le dommage provoqué par sa conduite. Lombroso admet l'efficacité intimidante de la peine en tant que sanction afflictive, dans le cadre d'ensemble d'un schéma de prévention, mais il ne la considère pas comme un élément décisif, et de plus il exclut la possibilité qu'elle résulte obligatoirement d'un châtiment infligé à la désobéissance. A la base des sanctions criminelles, envisagées comme des mesures préventives de défense sociale, nous trouvons la responsabilité sociale que Lombroso veut substituer à la responsabilité individuelle. Le coupable n'est plus le simple auteur d'un délit déterminé à un moment déterminé, mais le possible auteur d'infractions pénales futures vis-à-vis duquel la société doit non pas appliquer la peine appropriée à l'offense, mais prendre des mesures préventives aptes à éviter toute récidive. Les peines ne peuvent plus être celles-là même appliquées jusqu'alors. Le but principal de l'anthropologie criminelle fait de la personnalité du criminel l'objet primaire et principal des règles de justice pénale au lieu de la gravité objective du crime.

Toutes ces idées trouvées chez Lombroso, et qui font de lui leur père spirituel, sont à partager avec (si ce n'est parfois à redonner à...) E. Ferri ou Garofalo. Les juristes, de ce qui formera le "trio italien", sont bien souvent à la base de ces conceptions pénales évoquées précédemment, et on peut penser qu' ils sont les “pères légitimes” (surtout Ferri) de théories que Lombroso reprendra à son compte, portera et diffusera car le plus "reconnu", sans doute, de tous les trois.

Enrico Ferri ${ }^{12}$, sociologue et avocat de l'école positive. Ferri est parmi les premiers à avoir eu une vision assez synthétique et élargie du problème criminel. Il se démarque de Lombroso sans le renier mais en développant sa pensée. Il ne rejette

12. E. Ferri naît en 1856 à Mantoue. Professeur de droit, avocat, parlementaire, il occupe la scène juridique avec une thèse retentissante en 1879 sur le libre arbitre (l'imputabilità umana e la negazione del liberio arbitro) et la parution de ses Nuovi orizzonti del diritto e della procedura penale en 1880 (La Sociologie criminelle - Ferri, 1905 - initialement “les nouveaux horizons du droit pénal"). Professeur à l'Université de Rome, il est auteur d’un projet de code pénal en 1921. Il devient également député radial, orateur socialiste et on le dit un "des chefs les plus intransigeants du parti”. Il est chargé de la direction de L'Avanti de 1900 à 1905. Il évoluera, adhèrera au parti fasciste et mourra à Rome en 1929. Il est un des piliers de L'Archivio du psichiatria fondé en 1880 et produit de nombreux écrits sur le phénomène criminel. Ferri pensait "qu' il était impossible d'expliquer scientifiquement le crime, comme d'ailleurs toute autre action humaine ou même animale, s'il n'est pas considéré comme le produit de telle ou telle constitution organique et psychique personnelle, qui agit dans tel ou tel milieu physique ou social". 
pas totalement l'hypothèse atavique, ni la folie morale. Celui pour qui le crime est à la fois biologique et sociologique. Homme des substitutifs pénaux, il veut mettre en lumière le rôle d'une société qui doit s'efforcer d'appliquer les moyens préventifs avant d'attendre que le mal soit fait, mais également s'interroger sur les pénalités et leur application, ainsi que sur la nécessité d'améliorer ou d'inventer de nouvelles pratiques pénales. La diversité des facteurs criminels et la destination qui en découle des différentes catégories de délinquants, détermine la variété des moyens défensifs contre le délit, que la "sociologie criminelle” indique à la société et qu'elle répartit ainsi: ce sont les moyens préventifs ou d'hygiène sociale qui tendent à empêcher que le délit lui-même existe; les moyens réparateurs ou de dédommagement civil; les moyens répressifs temporaires qui peuvent être quelques-uns de ceux de l'arsenal répressif existant, enfin les moyens éliminatoires par lesquels la société, après avoir constaté qu'un individu donné est impropre à la vie sociale, l'exclut.

Les substitutifs pénaux sont une innovation dans la pensée pénale, ils s'allient en cela à la question de la sentence indéterminée. Il faudrait donc l'emprisonnement à temps indéterminé. On pourrait fixer les peines avec maximum et admettre la révision périodique des sentences. L'irrationalité pour Ferri est de croire à l'autorité de la chose jugée, parce que les juges ne sont pas infaillibles (l'existence des erreurs judiciaires). A cet emprisonnement à temps indéterminé se joindrait une autre réforme: la suppression de l'isolement cellulaire diurne (l'isolement nocturne étant une nécessité inattaquable). La vie en commun avec le travail est ainsi un procédé excellent d'hygiène morale.

Ces conceptions très nouvelles découlent véritablement d'une réflexion différente sur le délinquant qui allait atteindre son apogée avec la doctrine de la symbiose.

\section{La symbiose du crime}

Depuis des siècles, le crime a toujours été envisagé, jugé, haï, frappé comme un acte de méchanceté. Avec la sociologie ou l'anthropologie criminelle, le crime s'apparente à un phénomène d'ordre naturel plus ou moins nuisible, plus ou moins pathologique. Si l'on envisage le crime de cette manière, les conclusions théoriques, pratiques, sont radicalement différentes. Dès lors, toute l'idée de responsabilité morale (reflet d'une méchanceté volontaire et libre) devient insoutenable et il ne reste que la responsabilité sociale du criminel vis-à-vis de la société. La peine cesse d'être la panacée universelle pour les crimes et à la dynamique violente de la répression, on substitue la dynamique (moins facile, plus utile pour les individus aussi bien que la collectivité) de l'élimination ou l'atténuation préventive et sociale des causes anthropologiques, telluriques et sociales de la criminalité. 
En fait les doctrines spécifiques à E. Ferri, basées plus précisément sur le traitement pénal du délinquant, atténuent la rigueur de la conception du criminel-né, mais sans jamais désavouer le fondement de ces théories. "L'école italienne" garde son unité et se groupe toujours autour de Lombroso. Des thèses semblent apparaître parfois comme opposées à celles soutenues par le Maître de l'Université de Turin, mais elles ne contestent jamais profondément celles qui existent. Ferri a moins insisté sur les facteurs biologiques et s'il a proclamé que sa doctrine était tout autre chose "qu'une alliance entre le droit pénal et l'anthropologie criminelle" (Ferri, 1893, p. 7), c'est qu'il s'était rendu compte des exagérations lombrosiennes mais il n'en fait pas moins une place très importante, aux facteurs anthropologiques ${ }^{13}$.

Ferri a beaucoup "zigzagué" avec l'anthropologie criminelle, il a toujours cherché une position médiane entre toutes les théories qui s'offraient à lui, sans jamais vouloir en rejeter, sans jamais vouloir choisir non plus... Il apparaît souvent lors de congrès et de manifestations comme celui qui "calme le jeu", un médiateur, le "diplomate" de l'anthropologie criminelle. Essentiellement éclectique, il cherche à concilier dans une formule les multiples théories en présence ${ }^{14}$ et s'appuie sur un esprit synthétique pour construire, comprendre et défendre les théories italiennes. Ferri a précisé, clarifié tout ce qui était lié au traitement pénal du délinquant. Garofalo (le dernier du trio) va apporter des réflexions juridiques aux théories positivistes.

\section{L'importance de la notion du crime}

Garofalo est celui qui va s'attacher à ce qu'est le crime pour comprendre le phénomène criminel. En cela, il est sans doute un des premiers à avoir suivi, cru en cette orientation, à avoir tenté de s'attacher à cette notion, fréquemment oubliée, ignorée des recherches “criminologiques”. Pour Garofalo, le caractère du criminel doit être exclusivement cherché dans le crime et il est l'auteur du concept de témibilité.

\section{Le diagnostic du criminel. Le concept de témibilité}

Dès 1890, Lombroso préconisait la nécessité d'un examen médico-psychologique du criminel. Garofalo insiste sur le caractère indispensable de cette "enquête" sur

13. Il semble qu'à la veille de sa mort, en 1928 , il appela de ses vœux une reprise du développement de cette science. Voir Bouzat (1957, p. 21).

14. Ses adversaires lui reprochent de communiquer sans attendre et de semer autour de lui tout ce qu'il pense, tout ce qu'il sent, tout ce qu'il sait (AAC, 1886, p. 73). 
le criminel ${ }^{15}$. "La vie antérieure du délinquant doit nous être connue et ses liaisons. Lâge du délinquant est la circonstance la plus importante, il faudra savoir ensuite quelle est sa famille, l'éducation qu'il a reçue, quelles ont été ses occupations, quel était le but qu'il poursuivait dans la vie." ${ }^{16}$

Garofalo pense qu'on peut exprimer un jugement de valeur sur le criminel et en tirer "un pronostic" qui s'exprime en termes d'hypothèse sur son comportement ultérieur. Le critère d'état dangereux servira de base au diagnostic et au pronostic. C'est une notion utilisée en psychiatrie dès le début du XIX ${ }^{\text {ème }}$ siècle, en France, avec la loi de 1838 qui donne au préfet le pouvoir d'ordonner le placement d'office dans un établissement psychiatrique et pour une durée indéterminée puisque la décision est révisée tous les six mois, de toute personne dont l'état mental compromet l'ordre public et la sûreté des personnes. Garofalo va transposer ce concept pour le criminel aux alentours de 1880, sous le nom de témibilité. Le concept de "témibilité, déjà contenu dans un article de Garofalo publié en octobre 1878 dans le Giornale napolitano di Filosofia e littere sous le titre "Studi recenti sulla penalita", est précisé par lui dans une étude paraissant à Naples en 1880: Di un criterio positivo della penalita et développé dans son ouvrage ${ }^{17}$. Le terme de témibilité désigne, selon Garofalo, la perversité constante et agissante du délinquant et la quantité de mal qu'on peut redouter de sa part en sa capacité criminelle, d'où les termes parallèles, périculosité, redoutabilité, dangerosité, état dangereux, Garofalo va élargir ce concept en voulant lui donner un sens plus constructif. L'État dangereux doit tenir compte de l'adaptabilité; la capacité criminelle du délinquant n'est pas le seul élément de l'état dangereux, il faut encore rechercher l'idonéité du coupable à la vie sociale dans les différents cas de délit. Il faut alors déterminer pour chaque délinquant le genre de frein adapté à la spécialité de sa nature ou encore l'obstacle capable d'éloigner le danger. Il convient de rechercher la possibilité d'adaptation du délinquant, c'est-à-dire les conditions du milieu dans lequel on peut présumer qu'il cessera d'être dangereux ${ }^{18}$.

Ainsi l'état dangereux est à la fois défini par la capacité criminelle et par la possibilité d'adaptation. Cette meilleure connaissance du criminel permettra d'anticiper le devenir et de tirer un pronostic permettant le choix d'une pénalité ajustée à cette personnalité, tout en gardant l'objectif de protéger la société contre le crime. Nous reviendrons sur ce concept et l'application qu'il a pu revêtir dans la quatrième par-

15. Voir Congrès Pénitentiaire Saint-Petersbourg, 1890, Rapport de Lombroso, "Conviendrait-il d'organiser l'enseignement de la science pénitentiaire ?", Actes du Congrès Pénitentiaire, 1890.

16. R. Garofalo, La criminologie, p. 329, cité par J. Pinatel, 1961, p. 119.

17. Pour cette partie, voir Pinatel, 1961, pp. 119-122.

18. L'ensemble des propos de Garofalo sont cités par J. Pinatel, 1961, p. 120. 
tie de notre travail. Garofalo a creusé un sillon parallèle à celui de Lombroso, mais il l'a creusé sur un autre terrain. Lombroso a mis à nu les anomalies anatomiques du criminel, Garofalo ses anomalies morales. Pour l'un l'anthropologie est surtout l'anthropométrie, pour l'autre elle est une théorie cérébrale.

Les conceptions de Garofalo comprennent deux parties distinctes. L'une aux contours indécis, fortement teintée d'idéalisme, qui relève davantage de la philosophie pénale que de l'anthropologie criminelle ${ }^{19}$; la seconde s'efforce de dégager ce que les recherches sur les criminels nous ont appris de suffisamment net et général, relativement à sa nature et à ses caractères. Garofalo personnifie avec Lombroso et Ferri la "trinité" des maîtres de l'école italienne. Tous trois représentent en se complétant les initiateurs de l'anthropologie criminelle, dans ses données générales. Ils forment le cœur de l'école italienne et se partagent la direction de l'Archivio de psichiatria. Une unité les rassemble, et c'est à ce bloc que vont se heurter les opposants à ces conceptions dont Colojanni est le porte-parole le plus fameux en Italie.

A la manière de Lombroso, pour comprendre le crime, étudions le criminel mais non pas la façon dont il a été élevé, ni son éducation, ni les circonstances de sa conduite... L'anthropologie criminelle étudie le délinquant à sa place naturelle, c'est-à-dire dans le domaine de la biologie et de la pathologie. Malgré l'ambiguïté de ces travaux et des critiques qui commencent à fuser, Lombroso restera présent et n'entraînera pas dans la tourmente l'anthropologie criminelle. La polémique va créer un regain dans cette toute jeune discipline et en ce sens, Lombroso a eu un rôle positif. Il a fait naître des discussions, créer les passions, provoquer les critiques, indiquant ainsi les difficultés à surmonter, les erreurs à éviter. Dans tous les cas, il a imposé l'étude du criminel, jusque-là négligée. Il est au fondement de ce courant d'idées sur le phénomène criminel. L'école italienne a construit un canevas de théories, sur lequel vont pouvoir se définir, se créer, en alliance ou en opposition surtout, d'autres écoles ou mouvements. C'est le cas du mouvement criminologique français rassemblé autour d'A. Lacassagne et des archives de l'anthropologie criminelle. L'école italienne, de par le caractère novateur de ces théories, ne laissa nullement le monde scientifique indifférent. Elle provoqua des réactions multiples, variées, en tout cas nombreuses. Elle fut l'origine de création de mouvements qui sur ce terrain voulaient affirmer leurs propres convictions, souvent en opposition face à celles de l'école lombrosienne.

19. Selon Tarde, on peut y entrevoir une sorte de plan des incriminations futures destinées toutefois à se simplifier, à se rectifier et surtout à se préciser encore. 
Alexandre Lacassagne, l'école lyonnaise et la criminologie française

L'école italienne suscita bien des polémiques, mais provoqua également dès sa création une première réaction d'enthousiasme quasi générale.

Après un temps de séduction réussie en France, ce n'est pas tant ce que Lombroso énonce sur la nature du criminel qui posera problème à certains savants français, que ce qu'il dit des causes de la criminalité. Beaucoup de ces Français croient en une explication sociologique, que Lombroso a par trop négligée à leur goût. La seule explication biologique n'est pas suffisante à leurs yeux. Soyons clairs, ils ne la nient pas pour autant. Ils pensent que Lombroso n'a pas fourni les preuves mettant en avant la prédominance de cette explication qu'il tient pour fondamentale. C'est en ce sens qu'A. Lacassagne interviendra au Premier congrès international d'anthropologie criminelle à Rome en 1885.

Lacassagne au premier congrès international d'anthropologie criminelle à Rome: un discours décisif

C'est au congrès international d'anthropologie criminelle à Rome en 1885, qu'A. Lacassagne fit "irruption" et s'affirma face à Lombroso. Ses prises de parole à ce congrès, son exposé marquent bien l'expression d'une volonté et d'une conviction, peut-être également d'une stratégie. Lacassagne s’attaque à l'atavisme, clé de voûte de tout le système comme "une exagération, une fausse interprétation"... et déconstruit peu à peu l'échafaudage italien.

Enfin, il avance son hypothèse... l'importance du milieu social, et défend avec ardeur ses hypothèses et celles de tout son entourage. Il a beau terminer ce discours en concluant: "qu’à notre époque il n'existe plus d'écoles. Il n’y a que la vérité: elle est à tous" (Lacassagne, 1885, p. 183). Lacassagne, en s'opposant à Lombroso, et en affirmant très fermement ses conceptions, a ouvert une brèche. Il a d'ailleurs tenté d'éviter le conflit, mais l'affronte quand il est là:

Je regrette d'avoir mal compris. Les idées que je viens d'émettre ne sont pas nouvelles. Dans les différents travaux que j’ai publiés depuis dix ans, on peut voir que le milieu social a été l'objet de mes études. J'ai constaté l'existence des deux facteurs reconnus de tous, mais je tiens à le redire, je crois que le facteur externe ou milieu social tend à prendre de plus en plus une véritable prépondérance. Je n'ai pas à me disculper d'avoir voulu en combattant des théories attaquer des hommes pour lesquels je professe la plus grande estime (Lacassagne, 1885 , p. 183). 
Dès lors, Lacassagne va intervenir très souvent ${ }^{20}$, mêlant sa voix et son opinion dans les débats jusque-là dominés par les théories et intervenants italiens. Lacassagne fut le seul à s'opposer aux thèses de Lombroso ${ }^{21}$, ainsi qu'aux thèses italiennes leur étant subordonnées.

Ne nous y trompons pas, malgré l'opposition vigoureuse et affirmée de Lacassagne, ce congrès assura le triomphe des idées de l'école italienne sur le plan international. Mais néanmoins, ce fut plus “qu’un coup d'épée dans l'eau” pour Lacassagne et ceux dont il était symboliquement le porte-parole... Un différend se précisait; plus loin, plus profond que la polémique, des convictions, des principes s'opposaient.

Deux tendances, deux mouvements, deux hommes... Lacasssagne, à ce congrès de Rome, a posé le premier jalon “officiel” contre l'école lombrosienne. Sans colère, sans passion excessive, Lacassagne créera une revue fédérant de multiples auteurs et articles et à la base d'un groupe est considéré comme le porte-parole de la France en ce domaine et qui représente l'orientation nationale dans les congrès internationaux.

A. Lacassagne personnifie ce mouvement et les conceptions qui s'en approchent seront restituées. Mais la complexité, voire l'indépendance des jugements selon les thèmes font souvent légions, et les positions évoquées seront multiples, plurielles comme l'est ce mouvement. Nous chercherons la part sociologique déjà largement revendiquée dans le projet de cette école.

\section{La dimension "sociologique" de Lacassagne}

Ainsi pour Lacassagne, le crime est sinon un déchet social, tout au moins un produit du "milieu social"22. Ce terme générique englobant l'ensemble des actions extérieures, climatiques, physiques, chimiques, doivent s'y ajouter les influence d'éducation et d'entourage susceptibles de provoquer l'éveil des tendances "criminelles" existant

20. À la suite de l'exposé du professeur de Turin, E. Morselli: "Le nombre de suicides augmente-t-il en raison inverse de celui des homicides ?", Lacassagne est ravi de ce rapport, mais ajoute: "En France, le milieu social a une grande importance pour l'interprétation des résultats statistiques". À propos d'une question de Frigerio et Lombroso: "De l'épilepsie et de la folie morale dans les prisons et les maisons d'aliénés", pour ceux-ci, il y a identité fondamentale entre la folie morale et la criminalité instinctive d'une part, et l'épilepsie de l'autre. Lacassagne fait remarquer que le mot d'épilepsie larvée n'est pas assez défini pour en faire l'équivalent de criminalité... "[...] Il y aurait un danger pour l'avenir de l'anthropologie à employer devant un jury ou les magistrats une comparaison ou des mots dont on n'apprécierait pas exactement la valeur."

21. A noter les propositions "originales" du Dr. Albrecht.

22. D'où le nom donné à l'école française: école du "milieu social". Ce concept de milieu aura au cours du XIX ${ }^{\text {ème }}$ siècle deux nouveaux domaines d'application. En biologie, sous l'impulsion de Geoffroy Saint-Hilaire; pour les sciences morales, A. Comte et Taine (ces deux derniers ayant eu une influence très nette sur $\mathrm{A}$. Lacassagne). 
à l'état latent chez les individus "héréditairement tarés", ou les créer chez les sujets "normaux". Si le facteur individuel est dans certains cas prédominant, et si le côté pathologique s'accuse à tel point que son évidence soit manifeste, on a alors affaire à un fou et non à un criminel. "C'est la volonté accomplissant un acte et non l'acte lui-même qui fait le crime." (Laurent, 1890, p. 3).

Lacassagne ne croit pas au fatalisme, à la tare originelle.

Nous pouvons donc définir le crime: tout acte nuisible à l'existence d'une collectivité humaine. Nous savons encore que la personnalité morale se montre par des manifestations de sentiments, d'intelligence et d'activité. Depuis longtemps le langage vulgaire signale la prédominance de l'une ou l'autre de ces manifestations en reconnaissant des hommes d'esprit, de caractère, de cœur (Lacassagne, 1891, p. 406).

\section{Le milieu social, bouillon de culture de la criminalité}

On a dit que la société produisait des vertus et des vices comme elle fabrique du vitriol et du sucre. Lacassagne pense qu'il est plus exact d'avancer que comme la plupart des corps vivants, la société a ses parasites et ses microbes: ce sont les criminels. Une conception en somme très médicale, fortement influencée par les théories pasteuriennes, et qui tente d'adapter à la société "le corps", ces lois biologiques applicables à l'être humain:

De même le milieu social est le bouillon de culture de la criminalité. Le microbe est le criminel, un élément qui n'a d'importance que le jour où il trouve le bouillon qui le fait fermenter (Lacassagne, 1894, p. 407).

Ainsi le milieu social ou la société, par les insuffisances de son organisation politique, l'isolement et l'indifférence qu'elle peut engendrer, les sollicitations qu'elle multiplie, peut "encourager" la vocation criminelle. C'est l' inattention dont souffrent les plus défavorisés, exclus du bénéfice de l'éducation et souvent marginalisés par les mécanismes de l'économie moderne, qui les rend vulnérables aux sollicitations d'un environnement hostile. Ainsi, n'est-ce point avec le fatalisme qui découle des travaux de l'école italienne qu'il faut aborder la question du crime, mais avec toute l'imagination et l'initiative dont quelques-uns sont capables. Contre l'exercice systématique del'anthropométrie, il convient de promouvoir l'exemple, de développer l'éducation morale, de juguler les effets de l'industrialisme qui sacrifie l'homme à la marche du progrès. Si le milieu social est tout, et s' il est assez défectueux pour favoriser l'essor des natures vicieuses ou criminelles, c'est sur ce milieu et sur ses conditions de fonctionnement que doivent porter les réformes. 
Dans des travaux statistiques et spécialement dans un article de la Revue scientifique (Lacassagne, 1881), Lacassagne a minutieusement analysé les variations annuelles des crimes contre la propriété, les mettant au regard des oscillations de la situation économique, notamment du prix du blé.

Est-il nécessaire de souligner l'importance énorme de la thèse sociologique de l'état de délinquance, au point de vue de la lutte contre le crime? S'il était établi, comme le pensait Lacassagne, qu'en modifiant les facteurs criminogènes du milieu social, ses conditions hygiéniques et morales défavorables, on pouvait empêcher les prédispositions de naître chez les uns, ou de s'extérioriser chez les autres en réactions délictueuses ou criminelles, il serait relativement simple et aisé de prévenir l'éclosion et de combattre la récidive.

\section{Les aphorismes de Lacassagne}

Faites pour être dites, répétées, retenues, ces phrases sentencieuses qui résument en quelques mots ce qu'il y a de plus essentiel à connaître sur une question, (les aphorismes donc), vont être "le blason" de l'école lyonnaise.

A travers eux, Lacassagne montre en quoi le crime fait offense au progrès, comment il peut nuire à une collectivité, mais aussi comment il en relève les insuffisances. Ces paroles ont une double fonction, pédagogique et polémique. Les aphorismes doivent frapper; ils tiennent de la définition et du précepte. En eux se superposent la proposition dogmatique et la proposition pratique, la science et l'idéologie. On peut dénombrer six principaux aphorismes épars dans l'énoncé des théories de Lacassagne et de l'école française ${ }^{23}$ :

- le milieu social est le bouillon de culture de la criminalité, le microbe c'est le criminel, un élément qui n’a d'importance que le jour où il trouve le bouillon qui le fait fermenter,

- tout acte nuisible à l'existence d'une collectivité est un crime,

- tout crime est un obstacle au progrès,

- le criminel, avec ses caractères anthropométriques et autres, ne nous semble avoir qu’une importance bien médiocre, tous ces caractères peuvent se trouver d'ailleurs chez "d'honnêtes gens",

- au fatalisme qui découle inévitablement de la théorie anthropométrique, nous opposons l'initiative sociale,

23. A. Lacassagne, dans une de ses leçons, sur "Des transformations du droit pénal et les progrès de la médecine légale. 1810-1912”, AAC, 1913, cite ces aphorismes. 
- les sociétés n’ont que les criminels qu'elles méritent (ou là, c'est la société qui fait et prépare les criminels) (Lacassagne, 1913).

Il ne faut pas se fier à la trompeuse facilité de telles formules, qui se veulent être des "slogans" lapidaires, acérés, des instruments polémiques, des armes pour un combat d'idées.

Cette pensée ne reproduit pas toutes les nuances des aphorismes, qui sous leur apparente désinvolture révèlent, mettent l'accent sur différents problèmes; entre autres l'ambivalence que peut représenter le progrès: d'un côté utile, nécessaire, valeur de l'époque, dominé par l'élan scientifique qui amène un vent de liberté, un souffle d'espoir. De l'autre, "machine" par trop puissante, bouleversant les rythmes et les coutumes, accroissant la pauvreté, désemparant les plus faibles.

Quand Lacassagne parle d'industrialisme, ce n'est pas d'industrialisation, c'est l'énorme pouvoir "négatif" qui s'est dégagé du processus d'industrialisation, pouvoir en mouvement, force en croissance. Un "monstre" en somme dans cette difficulté, cette impossibilité de non maîtrise des événements et des phénomènes, l'aveu d'une impuissance devant un trop vaste et énorme processus; ces aphorismes respectent tous une éthique, un principe moral.

Ils traduisent également une ambiguïté dans la pensée de Lacassagne, valorise le progrès, tout en dénonçant certains effets pervers de phénomènes a priori dynamiques.

Aussi, sans oublier que c'est en réaction aux théories lombrosiennes que l'école française s'est affirmée, (ne niant pas la réalité biologique du phénomène criminel, mais en en refusant la prédominance ou l'exclusivité, et en introduisant la perspective “sociale"), elle est sortie de ce simple rôle d'adversaire, en se dotant d'une pensée plus large, plus nourrie. Une pensée qui ne s'est pas enfermée dans un simple dilemme de doctrines, mais s'est ouverte, s'est complexifiée et enrichie.

Déjà, la vision de l'homme criminel est l'image d'un être social dont l'histoire et les mouvements qui l'ont marqué sont à prendre en compte. "Le criminel n'est pas un sauvage, c'est l'homme moderne, produit de notre âge d'industrialisme et d'émancipation." (Lacassagne, 1909, p. 898).

Ainsi, l'homme n'est pas une entité abstraite et sa dimension sociale le caractérise.

Lacassagne a donc rendu possible et dirigé un mouvement qui s'est développé en parallèle avec celui de Lombroso mais qui, bénéficiant d'une "autonomie" et d'une ouverture totale, a transformé idéologiquement les questions du rapport du crime à la société et des facteurs de criminalité.

Les membres de l'école lyonnaise, partisans, élèves, collaborateurs ont peu à peu imposé la thèse du "milieu social", qui elle-même s'est affirmée sans problème 
dans l'environnement national, scientifique. Manouvrier nuancera et insistera sur l'ambivalence du milieu social, l'éducation en particulier qui pourra "rendre un loup non méchant", ou "rendre criminel un homme qui n'avait que quelques prédispositions” (Manouvrier, 1889, p. 561). Mais Lacassagne n'a pas de 1886 jusqu'en 1914 de véritables opposants en France. Des conceptions différentes, nuancées, apparaissent mais il n'émerge aucune thèse de la force de celle de l'École de Lyon. Les seules controverses de doctrine se jouent en marge (entre Tarde et Durkheim, par exemple) et n'engagent pas le sort de la théorie.

Aussi, de par cette relative unanimité, de par la force de la pensée et du dynamisme de son porte-parole, avec le flux des partisans, la naissance de l'école de Lyon, porte-parole officiel de la France dans les réunions internationales, correspond, représente la naissance d'une criminologie en France.

Les instruments ou espaces constitutifs du savoir: la Revue et les Congrès

La création des Archives Un instrument moteur: La Revue, une ville destinée: Lyon

Après l'éclat au congrès international de Rome, A. Lacassagne se démarque de l'école italienne, continue d'affirmer et son opposition et ses convictions en fondant une "école", mais et surtout un journal, parfait organe d'expression de ce mouvement français ou plus exactement "lyonnais".

Ainsi, avec les Archives de l'Anthropologie Criminelle, l'École française va naître, et alimenter une controverse de vingt-huit années (1886-1914) avec les Archives de Psychiatrie et d'Anthropologie Criminelle (1880-1917) de son homologue transalpine.

Cette lutte doctrinale n'est jamais véritablement meurtrière et n'exclut pas le dialogue constant (ou quasiment) qu'accompagnent néanmoins de profonds (ou moins profonds) différends.

En 1886, le journal paraît sous le titre:

Archives de l'Anthropologie Criminelle et des Sciences Pénales

Médecine Légale, Judiciaire - Statistique Criminelle - Législation et Droit.

L'anthropologie criminelle est dominante, les sciences pénales apparaissent en second plan. Ce titre subsistera jusqu'en 1893 où il se métamorphose, tout en accueillant à sa direction G. Tarde. Ce sont désormais:

Les Archives d'Anthropologie Criminelle, de Criminologie et de Psychologie Normale et Pathologique. 
De 1886 à 1893, trois directeurs se partagent la destinée de cette revue. A. Lacassagne, R. Garraud et H. Coutagne. En 1893, R. Garraud et H. Coutagne sont “relégués", ou plutôt transmettent leur pouvoir, et la "direction” est divisée entre A. Lacassagne pour la partie biologique, et là apparait sur la couverture mais surtout apparait sur la couverture en 1893.

Gabriel Tarde le "sociologue" de l'école française?

Cette direction sera assurée jusqu'en 1904, date du décès de G. Tarde ${ }^{24}$. Elle sera reprise pour la partie sociologique par P. Dubuisson. Cette alliance sera de courte durée, allant jusqu'en 1908, date de la mort de P. Dubuisson ${ }^{25}$. Dès lors, A. Lacassagne dirigera seul jusqu'en 1914. En cela il reste, de par sa continuelle présence, l'homme de la Direction.

Le Dr. Paul Dubuisson appartient à l'école positiviste ${ }^{26}$, et est décrit comme un “positiviste convaincu et pratiquant” (Lacassagne, 1908) qui réglait sa conduite privée et civique d'après les principes du maître. Il suivait avec assiduité les cours et conférences de Pierre Laffitte (professeur au Collège de France). En 1875-1876, il rédigera et publiera en deux volumes les leçons du successeur d'A. Comte sur Les grands types de l'bumanité.

\section{G. Tarde, "I'étoile filante"}

Collaborateur des Archives de l'Anthropologie Criminelle dès ses débuts, directeur pour la partie sociologique en 1893, G. Tarde est également un des auteurs les plus prolifiques de cette revue après Lacassagne, et ses articles paraissent de 1887 à $1901^{27}$.G. Tarde est un personnage dont le nom rehausse favorablement cette revue dont. Il y participe plus comme auteur que véritablement comme directeur. Il a laissé l'image d'un "marginal" aux idées originales, riches et fécondes, mais qui malgré cela, n’a jamais été à l'origine d'un mouvement particulier.

Il est sûrement plus créatif, plus "curieux" que Lacassagne, mais aussi plus personnel, plus “solitaire”. Il n’a pas créé, entraîné un mouvement et il n’a pas été éga-

24. Dès lors il sera fait mention de son nom sur les couvertures en dessous du titre: “[...] transformées en 1893 avec G. Tarde", AAC, 1905.

25. Son nom apparaîtra sur les couvertures à la suite de la mention de G. Tarde: “[...] transformées en 1893 avec G. Tarde et en 1904 avec P. Dubuisson”, AAC, 1909.

26. Sans doute est-ce à la Société positiviste de la rue Mr. le Prince que fréquentait Lacassagne (1874) qu'ils se sont tous deux rencontrés.

27. Bien quà cette date il soit décédé, il s'agit dès lors de publications posthumes. 
lement un "militant" d'un mouvement précis. Son appartenance à "l'école de Lyon" n'implique pas de sa part que cet engagement soit entier et absolu.

Les conceptions de Gabriel Tarde

Les théories de Tarde donnent aux facteurs psychosociaux et notamment à l'imitation un rôle important. Pour lui, la science criminelle ne doit pas être seulement une criminologie mais également une morale sociale.

\section{Une pensée antilombrosienne}

Tarde a fait d'abord le procès de la théorie du type lombrosien (ainsi que celle de la pluralité des types de Marro). Cependant, il ne niait pas qu'il "existe réellement un certain nombre de vrais criminels" mais leur crime restait, malgré tout, facteur du milieu. Tarde complète sa théorie du criminel par une théorie du crime qui s'inspire des mêmes données fondamentales, c'est-à-dire les données sociologiques. Il admet avec Ferril l'existence de trois catégories de causes en matière de criminalité, les causes physiques, physiologiques et sociales, mais reproche au maitre italien d'avoir fait la part trop belle aux causes naturelles alors que lui-même conclut à la prépondérance des causes sociologiques, avec un fond de toutes ces causes sociales, une notion précise: l'imitation, l'imitation liée à l'invention et le type professionnel.

Pour Tarde, l'idée du crime implique essentiellement, naturellement, celle d'un droit ou d'un devoir violé. Tarde part de l'observation suivante: le crime est un trouble social qui, partout et toujours, est caractérisé par une émotion qu' il suscite. C'est donc le critère de la violation par une volonté individuelle qui peut être retenu avec un dernier élément important aux yeux de Tarde: l'indignation morale. L'indignation fait partie de ces forces sociales qui établissent la dissidence entre un individu et son groupe. Elle réunit un consensus que toute la société ressent sur certains points regardés comme fondamentaux. Tarde en arrive à cette définition:

Le crime est une violation d'un droit, c'est-à-dire d'une volonté jugée supérieure (divine, royale, collective) contre laquelle s'est dressée une volonté rebelle et hostile; et cette violation est conçue comme présentant, à des degrés inégaux, ce double caractère d’être un danger social et une souillure sociale - un danger social à prévenir ou une souillure sociale à effacer. Comme un danger social, le crime est réprouvé par utilitarisme, comme souillure sociale par conformisme. Il alarme d'autant plus qu'il exprime chez son auteur une dissemblance morale plus profonde qui l'a rendu réfractaire à l'imitation morale de son milieu (Tarde, 1898, p. 350). 
"Le crime est un fait social comme un autre [...]" écrit Tarde, et en ce sens il est très proche de Durkheim et se distingue des écoles criminologiques, mais il séloigne de ce dernier quand il ajoute: "Mais un phénomène antisocial en même temps, comme un cancer, participe à la vie d'un organisme, mais en travaillant à sa mort... Le crime est une industrie, mais une industrie négative... (Tarde, 1898, p. 420$)^{28}$ :

Tarde reste autonome dans sa pensée malgré les influences lointaines qu’on peut lui retrouver ${ }^{29}$. Durkheim ${ }^{30}$ a fait beaucoup pour imposer et promouvoir la sociologie. Titulaire à Bordeaux de la première chaire française de sociologie puis professeur en Sorbonne, il va grouper autour de lui et surtout de sa revue (L'Année Sociologique fondée en 1896) une pléiade de chercheurs d'horizons très divers. Les idées de Durkheim sur le crime constituent une importante contribution pour la criminologie. Aussi il était difficile de ne pas les présenter même sommairement dans ce travail et la querelle avec Tarde sur ce sujet nous fournit le lien pour introduire de manière très “élaguée”, nous en convenons, la pensée de Durkheim sur ce sujet. L'idée maîtresse de Durkheim est de faire de la sociologie une science comme les autres, une science des choses. Cette science doit avoir un objet propre et une méthode objective. Pour cela et pour que la sociologie devienne une science autonome, il exclut l'intervention de la psychologie (devenue elle aussi une science autonome, distincte de la physiologie) dans l'étude des faits sociaux. La méthode doit être objective, scientifique; c'est-à-dire que l'observation des faits se conformera aux règles des sciences expérimentales, écartant toutes les pré-notions préexistantes dans l'esprit des observateurs.

Durkheim s'est surtout intéressé au suicide, considéré comme voisin de la criminalité à cette époque, mais il a été néanmoins soucieux d'établir en quoi le crime consiste essentiellement. Durkheim pense qu'il y a des crimes d'espèces différentes, mais que la réaction qu'ils déterminent est plus ou moins identique. L'acte le plus criminel n'est pas forcément le plus nuisible mais le plus contraire au dogme régnant, et en définitive le délit ne saurait être mieux défini que l'acte jugé délictueux par l'opinion publique. L'élément essentiel, ultime, que la sanction impliquée par lui ne fait que traduire, c'est la réprobation. Si bien qu' il ne faut pas dire qu'un acte froisse

28. Il précisait auparavant sa pensée (p. 366). Le crime est un fait social singulier, mais après tout un fait social comme un autre. Il est une branche gourmande de l'arbre national, mais une branche nourrie de sève commune et soumise aux lois communes.

29. E. Gillard (1921) parle de Montaigne, Fenelon et surtout Augustin Cournot.

30. Durkheim est néà Épinal en 1859, dans une famille juive. Patriote, il est meurtri par la défaite de 1870. Il poursuit une carrière universitaire très riche. La guerre de 1914 l'affecte beaucoup. Un de ses fils meurt en 1916. Lui-même décèdera en 1917. 
la conscience commune parce qu' il est criminel, mais qu'il est criminel parce qu' il froisse la conscience commune. Nous ne le réprouvons pas parce qu'il est un crime, mais il est un crime parce que nous le réprouvons. De toute façon, les crimes existent dans toutes les sociétés. La criminalité change de forme, mais elle existe partout et toujours ${ }^{31}$. Il n'y a donc pas de phénomène qui présente de manière aussi accentuée les symptômes de la normalité, puisqu' il est "lié" aux conditions d'existence de toute vie collective, donc le crime est un phénomène normal... Enfin, le crime a aussi une utilité directe. Le criminel doit être considéré comme un agent régulier de la vie sociale et non comme un mal. Quant à la peine, elle est le remède du crime, mais le crime n'étant pas une maladie, la peine ne saurait avoir pour objet de le guérir et sa vraie fonction doit être cherchée ailleurs.

\section{La "querelle" Tarde-Durkheim}

Ces conceptions très "modernes" émises principalement dans Les regles de la methode sociologique vont heurter G. Tarde. A première vue déjà, tout oppose ces deux hommes: le caractère, la carrière (exclusivement universitaire pour Durkheim), le projet, l'écriture même (la rigueur de Durkheim, l'échevelé de Tarde), plus leur système. Aussi est-ce peut-être pour cela qu'une polémique toute intellectuelle, semble-t-il, l'opposa à Durkheim, homme au caractère personnel et très indépendant également. Deux pensées originales, ardues vont débattre... Une polémique, (dont beaucoup pensent que Tarde est sorti vainqueur), s'instaure entre ces deux hommes, par revues et articles interposés. Ce ne sont pas les arguments de la logique de pensée qui sont mis au premier plan; ce débat porte sur des conclusions mais aussi sur les principes et la méthode... Et si l'objet central se trouve en être le crime, il est également le prétexte à une querelle plus méthodologique.

Tarde fait de l'imitation le fait social élémentaire, là où Durkheim voit dans la contrainte la marque propre de tout phénomène social, Tarde construit toute chose à partir de l'individu, quand Durkheim part de la société. Les convergences viennent de la conception que les deux "sociologues" se font des principes et de la logique sociologique. Le nœud de la controverse réside dans le caractère de normalité que Durkheim attache au crime, le distinguant du pathologique. Durkheim a attaché une grande importance à définir correctement le normal et le pathologique, la santé et la maladie. Durkheim entreprend d'établir que le crime n'est nullement un fait social pathologique. Le crime est normal parce qu'il n'est pas de société qui

31. "Partout et toujours, il y a eu des hommes qui se conduisaient de manière à attirer sur eux la répression pénale”, Durkheim, 1912, p. 82. 
ne le présente, parce que même une société qui en serait exempte reste du domaine de l'impossible. "Classer le crime parmi les phénomènes de sociologie normale, ce n'est pas seulement dire qu'il est un phénomène inévitable, quoique regrettable, dû à l'incorrigible méchanceté des hommes: c'est affirmer qu'il est un facteur de la santé publique, une partie intégrante de toute société saine” (Durkheim, 1912, p. 83). Quant au criminel, “contrairement aux idées courantes, le criminel n'apparaît plus comme un être insociable, comme une sorte d'élément parasitaire... C'est un agent régulier de la vie sociale" (Durkheim, 1912, p. 89). Durkheim fait chanceler une conception du crime propre à beaucoup de criminologues pour qui le caractère pathologique du crime est réel, incontestable ${ }^{32}$.

Tarde s'élève contre les conceptions de Durkheim; pour lui, ce dernier construit "en l'air, une sorte de sociologie en soi et pour soi qui, purgée de toute psychologie et de toute biologie, aurait bien de la peine à se tenir debout, sans le remarquable talent du constructeur" (Tarde, 1895, p. 148). Pour Tarde, le crime ne peut être un phénomène de normalité sociale parce que ce serait en pure contradiction avec le principe d'adaptation, fondement de la lutte pour la vie. Le crime comme la maladie peuvent permettre de vivre hors du combat, jamais d'y triompher. Tarde se demande si la constance d'un fait peut permettre d'aboutir, de penser qu'il est normal. Il répond négativement et malgré l'apparente logique du constat pense que Durkheim a seulement "exprimé avec beaucoup d'originalité une impression très banale, qui se traduit tous les jours par l' indulgence croissante des juges et jurés, par le relâchement des fibres de l'indignation et du mépris public en présence de certains attentats... J'ai bien peur pour M. Durkheim qu'il ne se trouve d'accord ici avec le sens commun ou plutôt vulgaire si méprisé par lui" (Tarde, 1895, p. 149).

Tarde tente de définir, de distinguer le normal de l'anormal. Il fait intervenir la notion de finalité et aboutit à une vision du normal pour la société, où on retrouve la paix, la justice, l'extermination du crime, du vice, de l' ignorance, de la misère, des abus. Tarde conteste également l'idée d'utilité du crime dont l'effet est d'atténuer ou même supprimer l'intérêt pratique de la peine. Tarde se demande à quoi peut être bon un crime? Dans un premier temps, à maintenir le niveau de conscience collective, répond Durkheim, car dans une société où il n'y aurait plus d'homicides, de vols, d'attentats aux mœurs, la conscience collective se mettrait à incriminer avec une sévérité extravagante les plus légers actes de violence. Pour Tarde cette justification est erronée car le péril actuel n'est pas à l'excès de scrupules de la conscience publique, ni la tendance à donner des pénalités démesurées pour des broutilles. Durkheim précise alors son

32. Bien que Durkheim ne remette pas en cause le fait que le criminel ne soit pas un individu normalement constitué. 
idée, reprochant à Tarde d'avoir exagéré celle-ci. Il ne pense pas que si certains crimes devenaient plus rares, les peines qui les frapperaient s'élèveraient nécessairement.

Il ne faut pas confondre répression et incrimination. De ce qu' ils seraient incriminés plus sévèrement, il ne suit pas qu'ils seraient punis plus sévèrement. Ce qui fait que ces deux ordres de faits ne varient pas l'un comme l'autre, c'est que très souvent, le sentiment collectif que le crime froisse est également froissé par la peine. Il s'établit ainsi une sorte de blâme. Le crime tout seul n'est pas à prendre en considération. Ce qui est normal, c'est le couple inséparable du crime et de la peine. Ainsi, voilà les points autour desquels Tarde s'est affronté très "courtoisement" à Durkheim... Points et conceptions très sommairement présentés mais permettant de mettre en rapport deux hommes d'envergure, deux pensées opposées.

Le premier a ouvert le chemin à la psychologie sociale, à l'étude des rapports sociaux et des formes de sociabilité, le second a permis le développement de l'étude des groupes sociaux et des institutions. Un niveau d'approche différent, des perspectives différentes. Chacun a une conception très personnelle de sa discipline, de sa méthode. C'est une des divergences entre ces deux hommes, conséquence de leur diversité conceptuelle ${ }^{33}$.J. Pinatel concluait pour une "fraternisation a posteriori" en écrivant: “il n'y a plus d'opposition entre la pensée de Durkheim et celle de Tarde. Le premier a été à l'avant-garde de la division du travail scientifique, tandis que le second a été le pionnier de sa réunification" (Pinatel, 1959, p. 442).

Ces conceptions de Tarde et de Durkheim, par leur caractère théorique et méthodologique, affinent et cisèlent une pensée qui reste dans la lignée des réflexions de Lacassagne et de sa revue.

Les Archives de l'Anthropologie Criminelle ${ }^{34}$, et le mouvement qu'elles rassemblent sont le centre moteur d'une réflexion sur la délinquance et le délinquant, sur le phénomène criminel dans ces années 1880 à 1914. Nos auteurs, par leurs études passionnées et leurs observations hétéroclites, par leurs analyses vivantes et complexes, bâtissent et sont à l'origine de ce savoir criminologique fondé sur l'homme. Diverses questions se posent: comment s'articule l'élaboration de ce savoir criminologique à travers la publication d'une revue lyonnaise et la naissance du champ intellectuel? (Charles, 1990; 1987; 1989).Comment un groupe d'individus "marginaux" par rapport à l'élite intellectuelle parisienne parvient à inventer une science, à faire croire à sa nécessité sociale? Quels sont les enjeux épistémologiques, ou autres, de

33. De Durkheim, Auguste Georges Berthier écrivait: "Sa confiance s'accompagnait, semble-t-il, du refus de tout crédit à une toute autre méthode de $s a$ méthode, à toute autre conception que sa conception..., "La Sociologie criminelle d'E. Durkheim", $A A C, 1914$, p. 317.

34. Nommé plus commodément, et en reprenant l'expression de l'époque, Les Archives (et dans les notes $A A C)$. 
l'affrontement qui oppose médecins et juristes dans la définition même de la criminologie? Comment se greffent là-dessus les enjeux nationaux qui transparaissent dans les polémiques des congrès?

Davantage que le contenu des articles publiés par la revue, ce qui semble important ce sont les nouvelles façons de penser qu' elle contribue à imposer par tout un travail de codification, de nomenclature, de structuration de la "matière criminelle". Des façons de penser qui peuvent finir parfois par se fixer et s' institutionnaliser dans les lois, des décrets, des politiques. Ces innovations conceptuelles finissent également par se transformer en pratiques sociales, d'abord hésitantes puis routinisées (ainsi de l'expertise médico-légale).

L'ensemble de ces éléments offre les conditions les plus favorables à la production d'un savoir riche et foisonnant sur le crime et le criminel.

\section{Droit pénal et sociologie criminelle}

Toutes ces innovations, tous ces bouleversements posent en effet le problème des rapports entre le droit pénal et la sociologie criminelle, et plus largement des influences des recherches anthropologiques et sociologiques sur le droit pénal. Le premier de ces thèmes a été l'objet de nombreux articles. Dès 1886, un des premiers articles des $A A C$, sous la plume de R. Garraud (1886), aborde cette question. Avant-première d'une revue qui débute, d'un mouvement qui se positionne, programme d'intentions et de principes où un bel hommage est rendu à Auguste Comte, fondateur de la philosophie positive qui le "premier a déterminé la véritable 'nature' des sciences sociales et la méthode qui doit être suivie dans leur étude". C'est une nécessité pour les sciences sociales "puisqu'il faut placer au sommet des connaissances humaines, non pour leur donner une vaine prééminence, mais parce que c'est en elle que s'opère la convergence de toutes les autres sciences" de s'appuyer sur les sciences naturelles et biologiques explique Garraud, et utiliser la méthode d'observation appliquée à leur étude est un élément d'importance pour les sciences pénales - droit pénal et sociologie criminelle - qui doivent apprendre à cohabiter.

Dans le droit pénal, le crime et la peine sont considérés comme des phénomènes juridiques, c'est-à-dire au point de vue des rapports des hommes entre eux et pour régler les droits et les obligations qui naissent de ces rapports. Rechercher quel est le fondement et quelles sont les limites du droit social de punir, se demander quels actes sont punissables, quelles sont les conditions de l'imputabilité et de la culpabilité, déterminer les conséquences du délit, soit au point de vue de l'intérêt privé, soit au point de vue de l'intérêt social, organiser des mesures de réparation et des mesures de répression. Tels sont, pour R. Garraud, les divers objets du droit pénal. 
Dans la sociologie criminelle, l'objectif change: le crime est considéré comme un phénomène social, "comme une maladie dont il importe de recherche les causes et de déterminer les remèdes", et la peine comme une fonction sociale. Tout en étant distinctes, ces deux manières de voir et de comprendre le crime et la peine doivent se pénétrer et réagir l'une sur l'autre. La sociologie criminelle peut donner une nouvelle orientation au droit pénal. Tel est le sens de l'article de Garraud en 1886; une vision harmonieuse et positive de ces deux domaines.

En 1893, Tarde évoquera à nouveau ces rapports:

La sociologie criminelle, pourrait-on dire avec assez de justesse, est au droit pénal ce que la physiologie pathologique est à la médecine. La sociologie en général, pourrait-on ajouter, est au droit ce que la physiologie est à l'hygiène, entendue dans le sens le plus large du mot, comme l'art de vivre sainement, dont l'art de guérir n’est qu'une partie (Tarde, 1893, p. 513).

En effet, pour Tarde, la législation, soit civile soit criminelle, est avant tout un art, et un art pour croître, peut et doit s'appuyer sur une science. Aussi: "La médecine n'est devenue un art national que le jour où elle a commencé à se fonder sur l'expérience scientifique, et le droit pénal n'a été vraiment digne du nom de Droit qu’à partir du moment où il a fait de la sociologie criminelle sans le savoir" (1893, p. 514).

Tarde tente de définir "la sociologie", parle des transformations du droit avec une grande lucidité, et surtout exprime toute sa confiance envers les vertus et qualités de la sociologie criminelle, bien différenciée à cet égard de l'anthropologie criminelle ${ }^{35}$.

Tous s'accordent sur la nécessaire entente entre sociologie criminelle et droit pénal.

Les médecins criminologues ont construit le crime comme objet scientifique digne de débats et de discussions. La somme de ces réflexions a poussé dans l'arène politique un objet qui par ailleurs devenait un objet public. Ce sont les juristes qui ici encore se réapproprieront par leurs débats, leurs modalités d'action, le crime et les enjeux pénaux qui s'y rattachent. C'est à l'intérieur, entre autres, de la Société générale des prisons, que vont s'élaborer les projets et politiques pénales républicaines.

35. Sur ce thème, R. Worms, directeur de la Revue Internationale de Sociologie, présente au congrès de l'Union internationale de Droit pénal (Paris, 1893) un rapport sur "Quelle influence les recherche anthropologiques et sociologiques peuvent-elles avoir sur le droit pénal ?” (Worms, 1893, pp. 673-677). Il estime que le Droit a une relation générale avec l'anthropologie, le Droit pénal une relation spéciale avec l'anthropologie criminelle, mais encore plus avec la sociologie criminelle. Car la distinction est nette entre les deux, l'anthropologie criminelle envisage l'homme criminel, la sociologie criminel le fait criminel. Où l'anthropologie criminelle considère le criminel en lui-même, en lui seul, abstraction faite du milieu qui l'entoure. La sociologie criminelle, pour Worms, au contraire essaie d'expliquer le crime par l'influence du milieu social dans lequel est plongé le criminel, étudie la réaction de la peine sur la société. 
La criminologie est difficile à cerner dans l'institutionnalisation de son enseignement, car il y est inclus de la médecine légale, des éléments de police technique, d'anthropologie de droit pénal. La création du certificat d'études pénales, par son programme, ses cours et travaux se rapproche bien des préoccupations d'une criminologie moderne. L'élaboration structurelle de la discipline se fera au travers des musées, de laboratoires et de la délivrance d'un enseignement, qui exprime une consécration de la discipline en formation. L'enseignement est la marque de l'institutionnalisation.

Il représente pour la discipline en question la reconnaissance du monde scientifique, et son entrée dans la sphère académique ${ }^{36}$. Très rapidement, des vœux sur l'enseignement de la médecine légale, des informations sur les établissements pénitentiaires pour les étudiants en droit ${ }^{37}$ ont vu le jour. En 1890 au congrès pénitentiaire international de Saint-Pétersbourg, Lombroso envoie un rapport intitulé: "Conviendrait-il d'organiser l'enseignement de la science pénitentiaire” et il place à l'intérieur d'un cours d'instruction pénitentiaire une partie théorique sur les lois, ordonnances et règlements, une étude de la statistique criminelle, les théories pénales, la libération conditionnelle et le patronage ainsi que des études d'anthropologie criminelle et de psychiatrie sur les criminels ${ }^{38}$.

36. Henry Joly écrivait: "La tendance de toute science est de se répandre et, par conséquent, de s'enseigner. Toute science, il est vrai, commence par une période dans laquelle elle ne doit rien quà l'initiative et aux efforts des travailleurs individuels. Tout savant qui a une idée tend à la propager. On ne peut cependant appeler du nom d'enseignement les appels intermittents et isolés que certains penseurs adressent d'abord à une partie restreinte du public, lorsqu' ils veulent substituer à l'ignorance ou à l'empirisme un ensemble de réflexions plus ou moins bien coordonnées. Mais quand ces réflexions se sont multipliées, quand elles ont éveillé l'opinion et provoqué des controverses, quand ceux qui discutent se sont mis d'accord sur quelques points, quand ils ont arrêté pour un temps la nature et le nombre des problèmes à résoudre, alors les esprits sont mûrs pour réclamer et pour accueillir un véritable enseignement" (Joly, 1890, II, p. 459).

37. On se rappelle le vœu de Lacassagne en 1889 , ou de Tarde, qui bien qu'absent, soutenu par Ferri et Aguglia, estime que les étudiants ne devraient être admis aux cours de droit criminel, de psychiatrie, qu'à la condition de se faire préalablement inscrire comme membres d'une société de patronage des prisonniers, présidée par leur professeur. Ainsi, ils seraient astreints à des visites en prison, et pourraient connaître les délinquants et criminels, en même temps qu'ils "pratiqueraient et propageraient" un des remèdes les plus efficaces contre le fléau de la récidive. Les autres congrès A. C. ont souvent rappelé ou mentionné ces questions (par exemple, en 1906 ou 1911).

38. En 1895, le congrès de l'Union internationale de droit pénal, votait la proposition suivante: "Afin que les criminalistes soient mieux formés et préparés à exercer leurs fonctions, il est désirable qu'ils ne soient pas instruits seulement du texte des lois pénales; il est à souhaiter que, soit par des cours facultatifs pour les étudiants, soit par des cours spéciaux destinés aux jeunes praticiens juristes, des notions plus étendues et plus approfondies leur soient données 
La criminologie, un enseignement juridique

Ainsi, la criminologie a réussi à trouver refuge... chez les juristes. L’approche reste multidisciplinaire, n'oubliant pas la médecine légale et la psychiatrie, mais reléguant "l'anthropologie criminelle". L'enseignement réussit à prendre forme en faculté de droit. On est passé d'une anthropologie criminelle, empirique et foisonnante, à une "criminologie" stabilisée et structurée en matières et théories. Le droit a récupéré ce savoir, qui en s'institutionnalisant par l'enseignement, s'imprègne d'un caractère de légitimité qui ne permet pas les énoncés des premiers travaux d'anthropologie criminelle, réalisés à partir d'une approche médicale. A travers cet enseignement, on a le sentiment d'un passage de la médecine légale aux études pénales, d’un regard médical à la règle juridique, de l'anthropologie criminelle à la criminologie. Très vite, l'anthropologie a été éloignée de la construction institutionnelle du savoir, très vite ce discours riche, excessif, outré, s'intéressant à l'homme (criminel) en s'académisant, se réduit, se spécialise, s'intéressant plus au criminel (homme), au hors-la-loi. De l'un à l'autre, il y a transformation et maîtrise d'un savoir en ébullition et une métamorphose de l'objet.

C'est le passage d'une science sociale en balbutiement à une science juridique qui s'affirme. L'ère de "l'anthropologie criminelle" telle que les congrès ont pu la représenter s'achève. La criminologie se stabilise, s'assagit et se légitime, en même temps que le grand mouvement international et national qui l'avait propulsée sous le nom d'anthropologie criminelle s'éteint avec la première guerre mondiale. Celle-ci ne faisant que mettre un coup fatal à ce qu'on pourrait analyser comme un essoufflement des idées du mouvement d'anthropologie criminelle. Il faudra attendre une vingtaine d'années pour que le problème de la criminologie soit à nouveau posé sur le plan international ${ }^{39}$.

Ce discours médical en s'institutionnalisant devient un objet juridique alors que ses fondements devaient beaucoup à l'analyse sociologique. Une analyse sociologique très tâtonnante reposant sur l'anthropologie, savoir lui-même en construction mais qui relève d'une première forme de science sociale.

sur les causes générales du crime, les particularités du monde des malfaiteurs et sur les procédés pratiques les meilleurs à suivre, dans la recherche des infractions et l'application des peines". Ce vœu aboutit à la création à Vienne d'un cours facultatif de criminalistique pour les étudiants en droit, mais on n'en tint pas compte dans les pays latins. AAC, 1904, p. 271.

39. C'est en 1934 que Benigno di Tullio créera la Société internationale de criminologie. Le premier congrès international de criminologie aura lieu à Rome en 1937 et en ce qui concerne les congrès nationaux, le premier congrès national italien de criminologie se déroulera à Vérone les 17-19 octobre 1959, fêtant le cinquantième anniversaire de la mort de Lombroso. Le premier congrès national français de criminologie se passera à Lyon en 1961 et un hommage prononcé sera rendu à Lacassagne. 
Ce fondement anthropologique restera le socle (mouvant mais présent) d'une sociologie qui se démarquera de plus en plus, s'affirmera et s'autonomisera totalement.

La criminologie, malgré ses voyages, ses transformations, ses évolutions gardera toujours en elle cette empreinte originelle autour de la naturalisation; la naturalisation du regard, de l'approche, amenant à des conclusions, qui aujourd' hui (pour certains) peuvent s'appuyer, sans complexe sur les neurosciences ou la psychologie, et renouer ainsi, en quelque sorte avec les débuts de ce savoir qui a toujours tenté de percer l'énigme à jamais irrésolue du mystère du passage à l'acte.

Ainsi, sur ce terrain du crime s'esquisse un partage de terrains entre médecins et juristes (Kaluszynski, 1995, pp. 215-235). Enjeux scientifiques, enjeux de compétences et peut-être enjeux politiques, semblent se mêler pour expliquer cette "fracture" qui maintient néanmoins les juristes en position de force. En 2009, une chaire de criminologie est créée au Conservatoire National des Arts et Métiers, en 2010 un rapport publié par la Conférence Nationale de criminologie propose un état des lieux de l'enseignement et formule des recommandations. Le 21 mars 2012, à la suite de la création par arrêté ministériel du 13 février 2012 d'une section CNU 75, criminologie, une motion est adoptée qui dénie toute légitimité scientifique à cette nouvelle section. A la suite du changement gouvernemental, celle-ci sera finalement supprimée par arrêté du 6 août 2012.

Il faudrait s' interroger et travailler sur cet "échec" français, là où existe un ancrage très fort, très cohérent (et donc réussi) en Belgique ou au Canada, d'une criminologie reconnue, validée, légitime. Celle-ci donne lieu à de travaux et des recherches de grande qualité (proches de la sociologie pénale ou de la sociologie du droit) portant avec noblesse l'étiquette de criminologie et ces auteurs identifiés et reconnus comme criminologues, sans contestation aucune.

\section{Références}

AAC - Archives de l'Anthropologie Criminelle et des Sciences Pénales: Médicine Légale, Judiciaire:

Statistique Criminelle: Législation et Droit. (1886-1892). Paris, Masson. AAC - Archives de l'Anthropologie Criminelle, de Criminologie et de Psychologie Normale et Pa-

thologique. (1893-1907). Lyon, Storck/Paris, Masson. AAC - Archives d'Anthropologie Criminelle, de Médécine Légale et de Psychologie Normale et Pathologique. (1908-1914). Lyon, Rey/Paris, Masson. ACTES du Congrès Pénitentiaire International de Saint-Petersbourg, 1890. (1890-1892), Saint-

-Petersbourg, Bureau de la Commission d'Organisation du Congrès. Ancel, M. (1978), “Le centenaire de L'Uomo Delinquente”. Revue Science Criminelle, I. 
Bouzat, P. (1957), “Le centenaire d'E. Ferri. L’œuvre du maître, son actualité”. Revue Science Criminelle.

Charles, C. (1987), Les élites de la République, 1880-1900. Paris, Fayard.

Charles, C. (1989), “Pour une histoire sociale des professions juridiques contemporaines”. Note pour une recherche dans Actes de la Recherche en Sciences Sociales, Droit et Expertise, 76-77.

Charles, C. (1990), Naissance des “intellectuels”, 1880-1900. Paris, Minuit.

Dammame, D. (1995), "Entre science et politique, la première science sociale, frontières disciplinaires". Politix, 29: 5-31.

Durkheim, E. (1912), Les règles de la méthode sociologique. Paris, Félix Alcan.

FERrI, E. ([1893] 1905), La sociologie criminelle. Paris, F. Alcan.

Foucault, M. (1975), Surveiller et punir, naissance de la prison. Paris, Gallimard.

Galton, F. (1878), "Les portraits composites", Revue Scientifique.

Garraud, R. (1886), "Rapports du droit pénal et de la sociologie criminelle”. AAC, Archives de l'Anthropologie Criminelle.

Gillard, E. (1921), “G. Tarde, sa métaphysique, sa sociologie, sa criminologie”. Revue de Droit Pénal de Criminologie.

Joly, H. (1890), "Rapport au congrès pénitentiaire international de Saint-Petersbourg”. Actes du Congrès, II.

Kaluszynski, M. (1995), “Identités professionnelles, identités politiques: médecins et juristes face au crime en France à la fin du XIX ${ }^{\text {ème }}$ siècle”. In: BlanckaerT, C. \& Mucchielli, L. (dir.). Histoire de la criminologie française. Paris, L'Harmattan, collection Histoire des Sciences Humaines, pp. 215-235.

Kaluszynski, M. (2002), La République à l'épreuve du crime: la construction du crime comme objet politique, 1880-1920. Paris, L. G. D. J.

Lacassagne, A. (1881), "La marche de la criminalité en France”, 1825-1880. Revue Scientifique, 28 mai.

LaCAssagne, A. (1885), "Congrès international AC”. AAC, Archives de l'Anthropologie Criminelle.

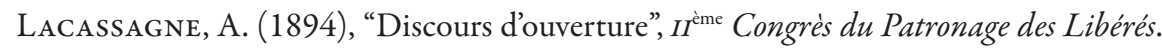

Lacassagne, A. (1908), "Notice nécrologique, sur Dubuisson”. AAC, Archives de l'Anthropologie Criminelle.

Lacassagne, A. (1909), “Gabriel Tarde”, Discours à l'inauguration de son monument. AAC, Archives de l'Anthropologie Criminelle.

LaCAssagne, A. (1913), "Des transformations du droit pénal et les progrès de la médecine légale. 1810-1912". AAC, Archives de l'Anthropologie Criminelle.

Laurent, E. (1890), Les habitués des prisons de Paris. Paris, Storck.

Leclerc, G. (1979), L’observation de l'homme. Une histoire des enquêtes sociales. Paris, Le Seuil.

Lombroso, C. (1887), L'Homme criminel. Paris, F. Alcan.

Lombroso, C. (1899), Le crime, causes et remèdes. Paris, Schleicher. 
Locard, E. (1907), "Revue critique”. AAC - Archives de l'Anthropologie Criminelle.

Manouvrier, L. (1889), “II ${ }^{\text {ìme }}$ Congrès international AC”. AAC - Archives de l'Anthropologie Criminelle.

Manouvrier, L. (1903), “Linnéité criminelle”, conférence posthume.

Perrot, M. (1975), "Délinquance et système pénitentiaire en France au XIX ${ }^{\text {ème }}$ siècle”. Annales EsC, 30 (1): 67-91.

Perrot, M. (1976), "Premières mesures des faits sociaux, les débuts de la statistique criminelle en France, 1780-1830". In: Pour une histoire de la statistique, Vaucresson.

Perrot, M. \& Robert, Ph. (publié et commenté par) (1989), Compte général de l'administration de la justice criminelle en France pendant l'année 1880 et rapport relatif aux années 1826 à 1880. Genève-Paris, Slatkine Reprints.

Pinatel, J. (1959), “La pensée criminologique d'E. Durkheim et sa controverse avec Gabriel Tarde". Revue de Science Criminelle et de Droit Pénal Comparé.

Pinatel, J. (1961), Histoire des doctrines criminologiques. Paris, Puf (Mélanges, Hommage à G. Heuyer).

Procacci, G. (1995), “La naissance d'une rationalité moderne de la pauvreté. L'Exclusion, l'Etat des savoirs. Paris, La Découverte.

TARde, G. (1890), Les lois de l'imitation. Paris, F. Alcan.

TARde, G. (1893), "La sociologie criminelle et le droit pénal”. AAC, Archives de l'Anthropologie Criminelle.

TARde, G. (1895), “Criminalité et santé sociale”. Revue Philosophique.

TARde, G. (1898), “Qu'est-ce que le crime?”. Revue Philosophique.

TARde, G. (1972), La philosophie pénale. 4 ème éd. Paris, Cujas.

Tort, P. (1985), “L'histoire naturelle du crime”. Le Genre Humain, Les usages de la nature. Paris, Complexe.

Worms, R. (1893),“Quelle influence les recherche anthropologiques et sociologiques peuvent-elles avoir sur le droit pénal?”. Rapport présenté au Congrès de l'Union internationale de Droit pénal de Paris. AAC, Archives de l'Anthropologie Criminelle. 


\section{Résume}

Entre science et politique, la criminologie, une science sociale en balbutiements...

La criminologie a aujourd'hui une actualité traversée par de nombreuses controverses, que ce soit sur son institutionnalisation, son développement dans le cadre d'un marché de la sécurité florissant, ou les emprunts qui y ont été faits lors de lois pénales assez récentes ${ }^{40}$. La criminologie a été abordée sous une forme picaresque et colorée or, il y a un vrai phénomène criminologie à analyser, tant cette "science sociale" relève des questions qui sont de réels problèmes de société. En s'attachant à la genèse de ce savoir, nous voudrions ici précisément montrer la dimension sociologique de ces écrits, proches de l'anthropologie, portés plutôt par des médecins, quelques juristes intéressés par le social et par l'impact de leurs travaux sur les possibles transformations du monde.

Mots-Clés: Science; Criminologie; Sociologie; France; Europe XIX ${ }^{\text {ème }}$ siècle; Politique; Savoirs de Gouvernement.

\section{Abstract}

Between science and politics, the criminology, a social science in its genesis...

Criminology today is riddled with much controversy, either because of its institutionalization, because of its development as part of a thriving security market or as loans that were made in very recent criminal laws. Criminology was approached in a picaresque and colorful way or as a way to address a real criminological phenomenon to be analyzed. In any case, this "social science" raises questions that are real problems for society. In focusing on the genesis of this knowledge, we would like here to show precisely the sociological dimension of these writings, close to anthropology, practiced mainly by doctors, some jurists interested in the social scope and the impact of their work on the possible transformations of the world.

Keywords: Science; Criminology; Sociology; France; Europe19 $9^{\text {th }}$ Century; Politics; Government Knowledges.

\section{Resumo}

Entre ciência e política, a criminologia, uma ciência social em suas origens...

A criminologia hoje é atravessada por muita controvérsia, seja em razão de sua institucionalização, em razão de seu desenvolvimento como parte de um próspero mercado de segurança ou de empréstimos que foram feitos em leis criminais bastante recentes. A criminologia foi abordada de forma picaresca e colorida ou como forma de abordar um fenômeno criminológico real a ser analisado. De todo modo essa "ciência social" levanta questões que são problemas reais da sociedade. Ao focarmos na gênese desse conhecimento, gostaríamos aqui de mostrar precisamente a

40. Loi renforçant la lutte contre la récidive des majeurs et des mineurs du 10 août 2007, appelée aussi loi sur la récidive ou loi Dati, ou dernièrement la loi relative à la rétention de sûreté et à la déclaration d'irresponsabilité pénale pour cause de trouble mental du 25 février 2008. 
dimensão sociológica desses escritos, próximos à antropologia, praticados sobretudo por médicos, alguns juristas interessados no âmbito social e no impacto de seus trabalhos sobre as possíveis transformações do mundo.

Palavras-chave: Ciência; Criminologia; Sociologia; França; Europa século XIX; Política; Saberes de Governo.

Texto recebido em 7/5/2020 e aprovado em 7/7/2020.

DOI: 10.11606/0103-2070.ts.2020.169496.

MARTINe KALUSZYNSKI é sócio-historiadora e diretora de pesquisas no CNRS (laboratoire Pacte UMr5194), professora no Instituto de Estudos Políticos de Grenoble, na França. Membro da direção editorial da revista Champ Pénal/Penal Field e redatora chefe da revista Criminocorpus. Seus trabalhos envolvem pesquisas sócio-históricas do Estado, das ciências de governo, do direito e da justiça. Autora de La République à l'épreuve du crime: la construction du crime comme objet politique, 1880-1920 (2002) e de várias pesquisas em colaboração com Sophie Wahnich, tais como L'Etat contre la politique? Les expressions historiques de l'étatisation (1998); com Olivier Ihl e Gilles Pollet, Les sciences de gouvernement (2003); com Jacques Commaille, La fonction politique de la justice (2007); com Renaud Payre, Savoirs de gouvernement, circulations, traductions, réceptions (2013); com Xavier Dupré de Boulois, Le droit en Révolutions. Regards sur la critique du droit des années 70 à nos jours (2011). E-mail: martine.kaluszynski@umrpacte.fr. 